Agronomie

\title{
Comparaison de 3 dispositifs expérimentaux utilisés pour estimer le rendement chez le blé tendre d'hiver (Triticum aestivum L.). Conséquence pour la sélection précoce
}

\author{
P. Brabant, A. Morin, E. Picard et M.-N. Mistou \\ avec la collaboration technique de J.-P. Meunier et $\mathrm{H}$. Corti
}

CNRS-INRA-UPS, ferme du Moulon, 91190 Gif-sur-Yvette, France

(reçu le 11 mai 1989, accepté le 20 septembre 1989)

\begin{abstract}
Résumé - Les rendements de 44 lignées ont été mesurés dans 3 dispositifs qui diffèrent principalement par la taille des parcelles élémentaires et par les densités de peuplement. Dans le dispositif "MR" la parcelle élémentaire est constituée d'un rang de $2,1 \mathrm{~m}$ de long et la densité de peuplement est de 175 plantes $\mathrm{m}^{-2}$. Le dispositif «PP» diffère du précédent par la taille de la parcelle élémentaire qui comporte 6 rangs. Le dispositif «GP» a des parcelles de 6 rangs de $4 \mathrm{~m}$ de long et le peuplement est de 250 plantes $\mathrm{m}^{-2}$. Ce dispositif est pris comme référence des conditions normales de culture de blé. Le dispositif MR est le moins précis, les corrélations trouvées avec GP montrent que la sélection précoce sur le rendement pratiquée sur MR correspond à une sélection indirecte. Un taux de sélection de $50 \%$ peut être pratiqué sur MR sans éliminer de très bonnes lignées. MR peut certainement être amélioré en éliminant les effets de bordure et en tenant compte de la compétition entre génotypes. PP est précis et en forte corrélation avec GP. Ce dispositif peut être utilisé pour étudier le rendement d'une collection de génotypes sur lesquels une information précise est recherchée.
\end{abstract}

sélection précoce - sélection indirecte - rendement - blé

Summary - Comparison of 3 experimental plots used to estimate yield in winter wheat (Triticum aestivum L.). Consequences for early selection. Yields of 44 lines were measured in 3 experiments which differed mainly in plot size and plant density. In the "MR" experiment, the plot consisted of a 2.1-m long a single row, with a density of 175 plants per square meter. The "PP" experiment differed from the previous one only by its 6-row plots. In the "GP" experiment, plots had 6 rows, $4 \mathrm{~m}$ long and plant density was 250 plants per square meter. GP was used as a reference for typical wheat farming conditions. MR appeared to be less precise; residual mean square estimates on $P P$ was 6 times higher than residual mean square estimates on PP and GP. The correlations that were found between yields measured on GP and MR and the estimation of genetic variance (Table IV) showed, that early selection carried out on MR would lead to indirect selection. Nevertheless, a selection rate of $50 \%$ could be carried out on MR results without eliminating good lines (Table 2 and Figure 1). MR could be improved by eliminating edging effects and taking into account competition between genotypes. PP was precise and highly correlated with GP (Tables I, III, IV and Figure 1). PP. can therefore be used to study precisely the yield of a collection of lines.

early selection - indirect selection - yield - wheat

\section{INTRODUCTION}

La sélection précoce comporte 2 aspects. Le premier, qui correspond à la notion de "selection in early generations" utilisée par Briggs \& Knowles (1967) cités par Weber (1984), désigne le fait de sélectionner des familles consanguines incomplètement fixées sur leur aptitude à donner des lignées pures de bonne valeur variétale (Gallais, 1978). Le deuxième, qui est celui auquel nous nous sommes intéressés, porte sur les dispositifs expérimentaux utilisables.

Au départ d'un schéma de sélection, la variabilité génétique est importante. Le grand nombre de familles soumises à la sélection nécessite la mise en place de dispositifs expérimentaux dans 
lesquels la parcelle élémentaire est de taille réduite. Chez les espèces à faible taux de multiplication, la quantité de grains disponibles par famille peut être le principal facteur limitant de la taille des parcelles. La sélection sur le rendement peut se pratiquer sur plantes isolées, mais avec une efficacité sujette à controverse qui, dans le meilleur des cas, reste faible (Mitchell et al., 1982). Dès que la quantité de graines disponibles par famille le permet, il est plus intéressant de mettre en place des microparcelles. Des dispositifs avec répétitions peuvent alors être envisagés. La parcelle élémentaire peut être semée en poquet (hill-plot), dispositif décrit par Bonnet \& Beaver (1947), ou bien semée en rangs (rowplot). Weber (1984) présente une revue des comparaisons entre les deux types de structures. Les corrélations sont, en général, positives et élevées.

Dans la sélection récurrente telle que nous la pratiquons sur blé tendre d'hiver, une sélection est pratiquée sur le rendement des familles $S_{1}$. Le dispositif comporte 2 blocs complets et la parcelle élémentaire est réduite à un seul rang. Nous avons trouvé dans ces conditions une héritabilité élevée pour le rendement (Brabant et al., 1989), mais comme le fait remarquer Weber (1984), il n'y a pas de nette différence entre sélection précoce et sélection indirecte. Dans le cas d'une sélection indirecte, le critère pertinent pour juger de l'efficacité de la sélection est le produit de l'héritabilité du caractère mesuré par le carré du coefficient de corrélation génétique avec le caractère que l'on souhaite améliorer (Hänsel, 1984). Dans l'étude que nous présentons, nous nous sommes intéressés à l'expression du rendement dans 2 dispositifs expérimentaux que nous utilisons en sélection. Le premier, dans lequel la parcelle élémentaire est monorang, est comparable à celui que nous utilisons en sélection récurrente. Le second est un dispositif où la parcelle élémentaire est formée de 6 rangs de faible longueur. Avec ce dispositif nous étudions la variabilité au sein de collections de lignées. Nous avons comparé les résultats obtenus dans ces dispositifs avec ceux obtenus dans un dispositif proche des conditions normales de culture du blé tendre d'hiver. Ceci, afin de juger si les dispositifs employés en sélection sont des facteurs perturbateurs suffisants pour nous placer en situation de sélection indirecte.

\section{MATÉRIEL ET MÉTHODE}

Le matériel végétal employé est constitué de 39 lignées pures et de 5 témoins. Les lignées ont été extraites par androgénèse d'une population à large base génétique nommée PB (Picard, 1984, Picard et al., 1988). Après une première année d'observation en pépinière en 1985/1986 les lignées ont été choisies sur des critères de taille et de résistances aux maladies parmi un effectif disponible de 220. Les 39 lignées retenues constituent un échantillon des lignées extractibles et potentiellement cultivables de PB. Les 5 variétés témoins sont: Arminda, Camp-remy, Festival, Fidel et Moulin.

Les 44 lignées ont été semées dans 3 dispositifs expérimentaux qui comportent chacun 3 blocs totalement randomisés. Les randomisations sont indépendantes entre blocs et entre essais. Le premier dispositif est constitué de parcelles élémentaires de 6 rangs de $4 \mathrm{~m}$ de long, ce dispositif sera par la suite appelé GP (Grande Parcelle). Le deuxième est constitué de parcelles de 6 rangs de $2,1 \mathrm{~m}$ de long. Nous l'appellerons PP (Petite Parcelle). Le troisième est constitué de parcelles d'un seul rang de $2,1 \mathrm{~m}$ de long. Nous l'appellerons MR (MonoRang). Ce dispositif (MR) comporte ici 3 blocs, alors que celui que nous utilisons en sélection précoce n'en comporte que 2. Le semis est effectué dans le sens des lignes avec un semoir porté par un tracteur. 6 rangs espacés de $23 \mathrm{~cm}$ sont semés à chaque passage. Entre chaque unité de 6 rangs il y a donc un passage de roue de tracteur sur un interrang de $35 \mathrm{~cm}$. Les grandes et les petites parcelles sont donc bordées, de chaque côté, par un passage de tracteur. Dans les parcelles monorang 6 génotypes différents sont implantés. Par unité de 6 rangs il y a donc 2 génotypes qui bordent le passage de tracteur. La dose de semis a été ajustée en fonction des taux de germination pour atteindre un peuplement de 250 plantes $\mathrm{m}^{-2}$ pour le dispositif en grandes parcelles et de 175 plantes $\cdot \mathrm{m}^{-2}$ pour les 2 autres dispositifs. Les essais GP ont été semés le 14 octobre 1986 et les essais PP et MR le 3 novembre 1986. Les 3 essais ont été implantés dans le champ d'expérimentation du Moulon sur des parcelles contigües. La fumure a été la même pour les 3 essais, constituée d'un apport avant le semis de $150 \mathrm{~kg} / \mathrm{ha}$ de P205 et de K20 et d'une fumure azotée apportée sous forme d'ammonitrate $50 / 50$ à raison de $50 \mathrm{~kg} / \mathrm{ha}$ au tallage et de $110 \mathrm{~kg} / \mathrm{ha}$ à la montaison. La couverture fongicide et insecticide ainsi que les désherbages ont été identiques pour les 3 essais.

Les grains ont été récoltés et battus mécaniquement pour GP et PP. La récolte de MR a été faite manuellement. Les rendements (RDT) ont tous été convertis en quintaux par hectare ramenés à $15 \%$ d'humidité. Sur les essais GP la hauteur (HA) et la date d'épiaison (DE) ont été notées.

Les analyses de variances ont été faites sur chaque dispositif avec le modèle fixe classique à 2 facteurs (lignée et bloc). Les comparaisons de moyennes ont été faites avec le test de Duncan.

Les analyses de variances ont aussi été faites en éliminant les témoins avec le modèle mixte à deux facteurs :

$$
\mathrm{X}_{i j}=m+\mathrm{L}_{i}+\mathrm{b}_{j}+\mathrm{E}_{i j}
$$

$m$ moyenne générale de l'essai, $L_{i}$ effet aléatoire de la lignée $i$, de variance $V_{1}, b_{j}$ effet fixe du bloc $j, E_{i j}$ résiduelle aléatoire, de variance $V_{r}$.

Pour chaque essai nous avons estimé $V_{l}$. Les écarts types de l'erreur calculés sur chaque estimation l'ont été suivant la formule donnée par Becker (1984). Les héritabilités du rendement ont été estimées dans chaque dispositif et correspondent au rapport 
$V_{l} /\left(V_{1}+V_{r} / 3\right)$. Les intervalles de confiances ont été calculés suivant la méthode proposée par Knapp et al. (1985).

Les corrélations entre essais ont été abordées en utilisant le coefficient de corrélation de rang de Spearman et le coefficient de corrélation linéaire, calculés sur les moyennes des 3 répétitions. A partir du modèle mixte (sans les témoins), nous avons estimé les coefficients de corrélation génétique. Les écarts-types des erreurs associés aux coefficients de corrélation génétique ont été calculés suivant les formules établies par Mode \& Robinson (1959) et Scheinberg (1966) reprises par Becker (1984). Le calcul des corrélations génétiques a été fait sous l'hypothèse d'absence de covariances environnementales entre les essais. Cette hypothèse se justifie par les randomisations indépendantes.

\section{RÉSULTATS}

L'examen du Tableau I montre que le rendement moyen estimé dans le dispositif GP est inférieur aux rendements estimés dans les 2 autres dispositifs. Dans les 3 dispositifs l'effet lignée est très hautement significatif (la lignée $n^{\circ} 33$ très versée dans le dispositif GP n'a pas été récoltée ce qui explique dans ce dispositif les 42 degrés de liberté du facteur lignée). L'effet bloc n'est significatif au seuil de $5 \%$ que dans le dispositif PP. La variance résiduelle est estimée identique pour PP et GP, par contre elle est estimée 6 fois plus grande dans le dispositif MR.

Dans le Tableau II figurent les classements des lignées dans chaque dispositif. Les groupes de tête, homogènes au seuil de $1 \%$ pour le test de Duncan, sont figurés. Les classements obtenus dans les 3 dispositifs sont globalement concordants, comme l'indiquent dans le Tableau III les valeurs hautement significatives du coefficient de corrélation de rang de Spearman. Parmi les 20 meilleures lignées du dispositif MR (groupe de tête homogène au seuil 1\%) il en figure 15 et 16 parmi les 20 premières des dispositifs PP et GP respectivement. Les corrélations linéaires

Tableau I. Analyses de variances sur le rendement. Comparaison entre les dispositifs.

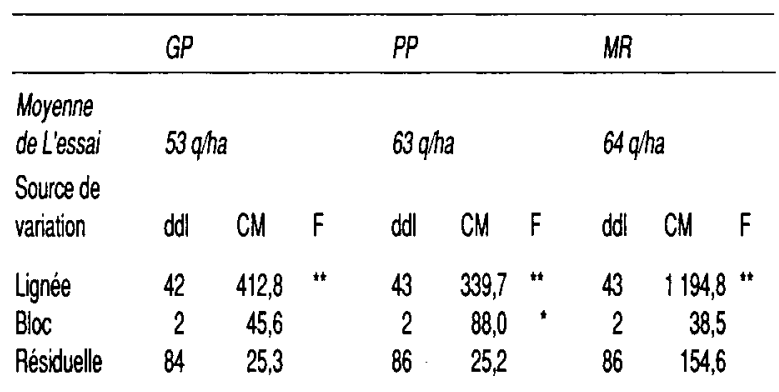

ddl : degrés de libertés; $\mathrm{CM}$ : carré moyen; ${ }^{* *}:$ significatif au seuil de

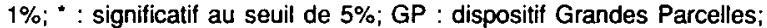
PP : dispositif Petites parcelles; MR : dispositif en parcelles Monorang.
Tableau. II. Classement des lignées suivant leur rendement moyen décroissant.

\begin{tabular}{|c|c|c|c|}
\hline Dispositi: & $G P$ & $\begin{array}{c}P P \\
\text { Numéro des lignées }\end{array}$ & $M R$ \\
\hline & $\begin{array}{l}14 \\
20 \\
44 \\
01 \\
29 \\
43 \\
18 \\
28 \\
02 \\
10 \\
04 \\
03 \\
25 \\
11 \\
06 \\
38 \\
34 \\
07 \\
35 \\
22 \\
40 \\
32 \\
05 \\
31 \\
19 \\
26 \\
16 \\
39 \\
24 \\
08 \\
41 \\
12 \\
37 \\
15 \\
42 \\
17 \\
27 \\
21 \\
09 \\
13 \\
36 \\
23 \\
30\end{array}$ & $\mid \begin{array}{l}29 \\
11 \\
44 \\
14 \\
34 \\
33 \\
43 \\
28 \\
20 \\
37 \\
01 \\
04 \\
03 \\
06 \\
13 \\
25 \\
02 \\
16 \\
18 \\
10 \\
38 \\
07 \\
12 \\
09 \\
35 \\
19 \\
39 \\
17 \\
24 \\
31 \\
41 \\
32 \\
26 \\
22 \\
15 \\
27 \\
42 \\
05 \\
08 \\
21 \\
40 \\
36 \\
23 \\
30\end{array}$ & $\begin{array}{l}16 \\
29 \\
03 \\
10 \\
44 \\
14 \\
01 \\
26 \\
09 \\
38 \\
28 \\
36 \\
04 \\
20 \\
34 \\
02 \\
06 \\
25 \\
11 \\
07 \\
33 \\
12 \\
24 \\
43 \\
41 \\
19 \\
35 \\
18 \\
27 \\
37 \\
13 \\
39 \\
17 \\
21 \\
22 \\
40 \\
15 \\
05 \\
30 \\
08 \\
23 \\
31 \\
32 \\
42\end{array}$ \\
\hline
\end{tabular}

: Groupe homogène des meilleures lignées, pour le test Duncan au seuil $1 \%$; les lignées 01 à 05 sont les variétés témoins.

Tableau III. Corrélations entre dispositifs.

\begin{tabular}{|c|c|c|c|c|c|c|c|c|}
\hline \multirow[b]{2}{*}{ GP } & \multicolumn{3}{|c|}{$G P$} & \multicolumn{3}{|l|}{$P P$} & \multicolumn{2}{|c|}{$M R$} \\
\hline & $\begin{array}{l}\text { is } \\
\text { r1 }\end{array}$ & $\begin{array}{l}1,00 \\
1,00\end{array}$ & ** & & & & & \\
\hline PP & is & 0,66 & "** & rs & 1,00 & & & \\
\hline & $\mathrm{r} 1$ & 0,80 & ** & $\mathrm{r1}$ & 1,00 & & & \\
\hline MR & $\begin{array}{l}r s \\
r 1\end{array}$ & $\begin{array}{l}0,54 \\
0,55\end{array}$ & "** & $\begin{array}{l}\text { rs } \\
\text { rt }\end{array}$ & $\begin{array}{l}0,66 \\
0,68\end{array}$ & 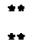 & $\begin{array}{l}\text { rs } \\
\text { r1 }\end{array}$ & $\begin{array}{l}1,00 \\
1,00\end{array}$ \\
\hline
\end{tabular}

rs : coefficient de corrélation de rang de Spearman; $r l$ : coefficient de corrélation linéaire; "* : significatif au seuil de $1 \%$; Pour n (nombre d'observations) supérieur à $30, r s \times(n-1)^{1 / 2}$ suit une loi normale centrée réduite.

(Tableau III) sont toutes très hautement significatives. La plus forte corrélation est obtenue entre GP et PP et la plus faible entre GP et MR. Les graphes de corrélations, représentés sur la Figure 1, illustrent la très bonne liaison entre GP et PP et les moins bonnes liaisons de MR avec GP et PP.

Les analyses de variances en modèle mixte, pratiquées sur les 38 lignées communes aux 3 dispositifs, permettent d'estimer les héritabilités, 

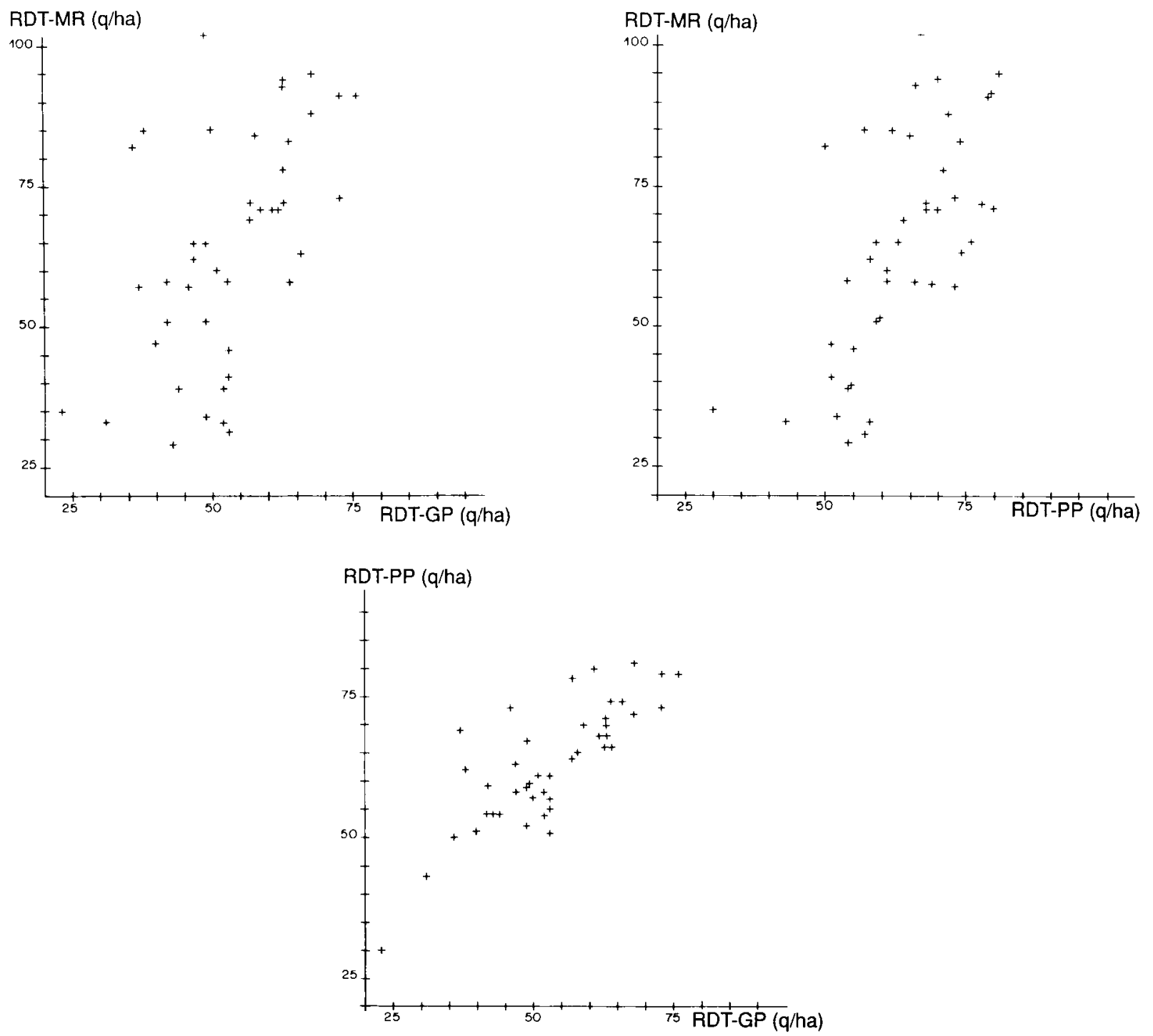

Fig. 1. Graphiques des corrélations entre les rendements mesurés dans les trois dispositifs.

les variances génétiques et les corrélations génétiques (Tableau IV). Les héritabilités sont estimées très fortes dans les 3 dispositifs. Les variances génétiques estimées dans GP et PP peuvent être considérées égales (la différence entre les 2 est inférieure à un écart type de l'erreur). Par contre la variance génétique estimée sur MR est environ 3 fois supérieure aux 2 autres et en diverge de 2,5 écarts types d'erreur. II paraît donc logique de rejeter l'hypothèse d'égalité de la variance génétique estimée sur MR avec celles estimées sur les 2 autres dispositifs.

Les corrélations phénotypiques estimées sur l'échantillon aléatoire de lignées (Tableau IV) sont très proches des corrélations linéaires du Tableau III. Les 5 témoins ne perturbent donc pas ces corrélations. La distribution de l'estimateur du coefficient de corrélation génétique est inconnu (Becker, 1984), il est donc difficile de raisonner à partir des écarts types de l'erreur. Toutefois, on remarque que les différences entre les
3 corrélations génétiques estimées et la valeur 1 sont de 2,6-3 et 3,5 écarts types de l'erreur pour les corrélations entre GP et PP, PP et MR, GP et MR respectivement.

Tableau IV. Paramètres estimés sur l'échantillon aléatoire de lignées.

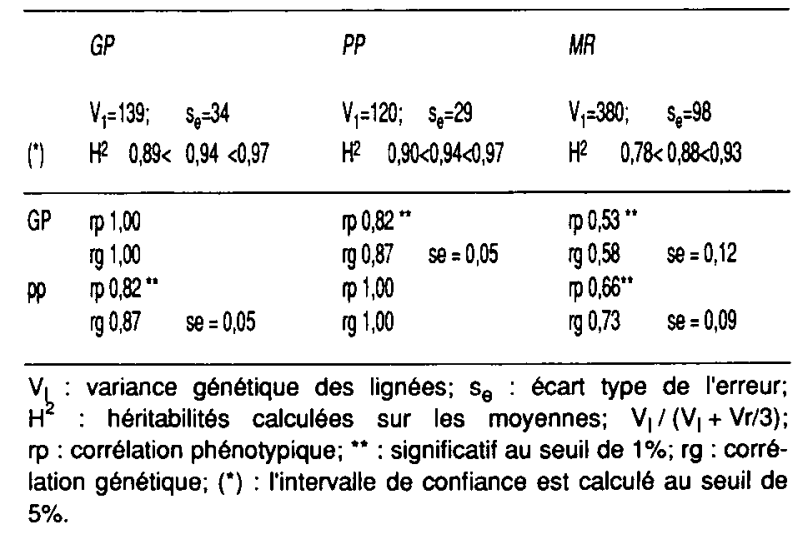




\section{DISCUSSION}

L'année 1987 a été particulièrement favorable à la verse sur les parcelles du Moulon. La plus forte densité de peuplement du dispositif GP a entraîné une verse plus précoce et plus forte sur ce dispositif, ce qui peut expliquer en partie les rendements moyens plus faibles de GP. Par ailleurs, les surfaces corrigées appliquées à chaque dispositif pour ramener les rendements en quintaux par hectare, ne prennent qu'imparfaitement en compte les effets de bordures. Ces effets sont d'autant plus importants que la parcelle est petite et peuvent expliquer une certaine surestimation des rendements PP et MR.

Pour comparer la précision des différents dispositifs nous pouvons utiliser la résiduelle des analyses de variances. Sur ce critère les dispositifs GP et PP sont équivalents et MR est beaucoup moins précis, puisque sa résiduelle est 6 fois plus grande. On peut aussi utiliser la statistique $\mathrm{F}$ de Fisher affectée au facteur lignée pour juger du pouvoir discriminant des différents dispositifs. Les valeurs trouvées sont 16,32, 13,48 et 7,73 pour GP, PP et MR respectivement. Sur ce critère GP apparaît légèrement plus performant que PP et MR reste le moins bon. Cette différence est illustrée (Tableau II) par la taille de plus en plus grande des groupes de tête, homogènes au seuil $1 \%$ pour le test de Duncan. Dans la suite nous prendrons comme référence les résultats obtenus sur GP.

L'examen des corrélations (Tableau III) montre qu'aussi bien pour les corrélations de rang que pour les corrélations linéaires, les dispositifs GP et MR sont les plus différents. Les corrélations linéaires et les graphes de la Figure 1 montrent que PP est très proche de GP et qu'il existe une différence plus marquée entre PP et MR.

En conclusion sur l'analyse en modèle fixe, nous dirons que les lignées vues mauvaises dans le dispositif MR sont moyennes ou mauvaises dans les 2 autres dispositifs (Fig. 1). MR apparaît donc efficace pour éliminer des lignées peu performantes dans du matériel peu sélectionné. Un taux de sélection de $50 \%$ pratiqué sur ce dispositif, n'élimine pas de lignées jugées bonnes dans les 2 autres dispositifs. Le dispositif PP pourrait être amélioré en augmentant la densité de peuplement pour se rapprocher des conditions normales de culture. PP présente 2 avantages par rapport à GP. Pour un même nombre de génotypes testés, la surface occupée sur le terrain est d'un tiers plus faible et le temps de récolte est lui aussi réduit d'un tiers. Ce dispositif nous paraît tout à fait performant et très utile en sélection pour trier de bonnes lignées parmi une vaste collection. Cette situation peut se ren- contrer en sortie de Bulk de SSD ou d'haplodiploïdisation.

Les résultats obtenus sur l'échantillon aléatoire de lignées font apparaître de fortes héritabilités pour le rendement dans les 3 dispositifs. Ces héritabilités s'expliquent par la forte diversité génétique des lignées testées et, pour GP et PP par une bonne qualité des essais (faible résiduelle). L'essai MR surestime fortement la variance génétique, ce qui explique la forte héritabilité trouvée dans ce dispositif. Les corrélations génétiques confirment la proximité de GP et de PP et la divergence entre GP et MR. Que ce soit pour PP ou pour MR, il paraît difficile d'admettre qu'on se trouve en situation de sélection directe (le rendement mesuré sur GP étant pris comme référence). La valeur de 0,87 trouvée pour la corrélation entre RDT-GP et RDT-PP est toutefois très élevée. L'écart à la valeur 1 peut s'expliquer par les différences de date et de densité de semis des 2 dispositifs. Ces 2 facteurs peuvent aussi expliquer la plus grande proximité entre $\mathrm{PP}$ et MR qu'entre GP et MR. Pour expliquer la divergence de MR par rapport aux 2 autres dispositifs nous invoquerons 2 facteurs de perturbation :

- un tiers des lignes du dispositif MR borde un passage de tracteur;

- il y a de la compétition entre les lignées à cause du faible espacement entre les rangs. Des observations faites la même année sur d'autres essais (Mistou, non publié) montrent que le fait d'occuper une ligne de bordure conduit à une surestimation du rendement de l'ordre de $11 \%$. Une amélioration possible du dispositif MR serait de supprimer la discontinuité introduite par le passage du tracteur. Deux solutions sont possibles, semer perpendiculairement au sens des lignes ce qui nécessite un équipement spécial ou border les passages avec une variété tampon ce qui fait perdre un tiers de la surface. La compétition entre génotypes est un problème moins facilement soluble. Fasoulas (1984) passe en revue les effets de la compétition intergénotype sur l'expression du rendement. II en conclut qu'en sélection précoce, sous-entendu dans des familles non fixées, il faut sélectionner sur plantes isolées. Dans la situation que nous décrivons, l'isolement entre plantes ou même entre rangs ne nous paraît pas une bonne solution. En effet, dans les conditions normales de culture d'une variété de blé, la compétition intervient sur le rang entre les plantes et entre les rangs. Sneep (1976) préconise l'emploi de parcelles de 3 rangs dont seul le rang central est récolté. Notre pratique de l'expérimentation sur le blé nous conduit à penser que la récolte globale des 3 rangs est plus informative que la récolte du seul rang central. De plus, ce type de dispositif 
est très consommateur de semences. Nous pensons qu'il serait plus intéressant d'utiliser les voisinages entre parcelles pour minimiser les effets de la compétition intergénotypique. Deux approches nous paraissent directement utilisables. L'utilisation de covariables ou l'estimation directe des effets producteur et compétiteur de chaque génotype (Azaïs et al., 1984; Speckel et al., 1987).

Nous possédions les mesures faites sur GP, de la hauteur (HA) et de la date d'épiaison (DE) des lignées. Ces 2 caractères nous ont paru intéressants à utiliser en régression linéaire pour comparer leur capacité à expliquer le rendement dans les différents dispositifs. Notre idée était qu'en situation de compétition, une lignée haute et précoce est favorisée par rapport à une lignée courte et tardive. Le Tableau $V$ montre qu'effectivement $H A$ et $D E$ ont un pouvoir explicatif du rendement nettement supérieur dans le dispositif MR. Ces 2 variables sont faciles à mesurer et nous paraissent intéressantes à prendre en compte comme covariables.

Tableau V. Explication du rendement par la hauteur (HA) et la date d'épiaison (DE).

\begin{tabular}{llll}
\hline $\begin{array}{l}\text { Corrélations entre les } \\
\text { variables }\end{array}$ & & $\begin{array}{l}\text { Coefficients de prédiction } \\
\text { obtenus par régression } \\
\text { linéaire } r^{2} \text { (HA, DE) }\end{array}$ \\
\hline & HA & DE & \\
RDT-GP & 0,274 & $-0,173$ & 0,104 \\
RDT-PP & $0,321^{*}$ & $-0,213$ & 0,147 \\
RDT-MR & $0,358^{*}$ & $-0,348^{*}$ & 0,247 \\
\hline
\end{tabular}

" : significatif au seuil $5 \%$ (ddl = 41 (GP), 42 (PP et MR)).

\section{CONCLUSION}

Le dispositif monorang que nous employons pour effectuer le tri des familles en sélection récurrente (Brabant et al., 1989) est imparfait à cause des effets de bordure et de la compétition entre rangs occupés par des génotypes différents. L'utilisation de variétés tampon permettra de minimiser l'effet de bordure. L'information fournie par les voisinages et des covariables devrait aussi permettre d'augmenter la fiabilité des essais monorangs. Un essai a été mis en place en 1988/1989 pour juger de l'intérêt relatif des différentes améliorations proposées.

La corrélation génétique de 0,58 entre les rendements mesurés sur GP et sur MR et la variance génétique estimée 3 fois plus grande dans MR, nous paraissent de bons arguments, pour retenir l'hypothèse selon laquelle, le dispositif MR nous place en situation de sélection indirecte par rapport aux conditions normales de culture, représentées par le dispositif GP. L'héritabilité mesurée dans le dispositif MR n'est donc pas le bon critère à prendre en compte pour juger de l'efficacité de la sélection pratiquée sur ce dispositif. II faut multiplier cette héritabilité par un coefficient, estimé ici à $0,34\left(0,58^{2}\right)$. Ce qui signifie qu'en terme de gain attendu après sélection on ne réalise que le tiers de ce que la simple observation de l'héritabilité laisse prévoir.

Le dispositif PP permet par rapport à GP, d'économiser de la semence, de la surface d'expérimentation et du temps à la récolte. Les résultats que nous obtenons montrent qu'il est précis et en bonne corrélation avec GP. PP est donc tout à fait utilisable pour étudier la diversité d'une collection de lignées et y effectuer un tri efficace sur le rendement.

\section{RÉFÉRENCES}

Azaïs J.M., Denis J.B. \& Kobilinsky A. (1984) Premières Idées sur la Compétition Interparcẹllaire. Publication interne, laboratoire de biométrie, INRA, 78000 Versailles, $20 p$

Becker W.A (1984) Manual of Quantitative Genetics, 4th edition. Academic entreprises, Pullman, Washington

Bonnet O.T. \& Beaver W.M (1947) Head-hill method of planting head selections of small grains. J. Am. Soc. Agron. 39, 442-445

Brabant P., Manes Y., Trottet M. \& Picard E. (1989) Corrélations génétiques, héritabilités et possibilités de sélection multilocale précoce sur le rendement chez le blé tendre d'hiver (Triticum aestivum L.). Agronomie 9, 49-54

Briggs F.N. \& Knowles P.F. (1967) Introduction to plant breeding. Reinold Publ. Corp. New York

Fasoulas A. (1984) Effects of competition in the selection process. In : Efficiency in Plant Breeding, Proceedings of the 10th Congress of Eucarpia (Lange W., Zeven A.C. \& Hogenboom N.G. eds.), Pudoc, Wageningen, 35-39

Gallais A. (1978) Amélioration des populations, méthodes de sélection et création de variétés. II. Le concept de valeur variétale et ses conséquences pour la sélection récurrente. Ann. Amélior. Plant. 28, 269287

Hänsel H. (1984) Selection for a complex characteristic by a substrate (Tables for indirect selection) In : Efficiency in Plant Breeding, Proceedings of the 10th Congress of Eucarpia (Lange W., Zeven A.C. \& Hogenboom N.G. eds), Pudoc, Wageningen 61-68

Knapp S.J., Stroup W.W. \& Ross W.M. (1985) Exact confidence intervals for heritability on a progeny mean basis. Crop Sci. 25, 192-194

Mitchell J.W., Backer R.J. \& Knott D.R. (1982) Evaluation of honeycomb selection for single plant yield in durum wheat. Crop. Sci. 22, 840-843 
Mode C.J. \& Robinson H.F. (1959) Pleiotropism and the genetic variance and covariance. Biometrics 15 , 518-537

Picard E. (1984) Contribution à l'Etude de l'Hérédité et de I'Utilisation en Sélection de I'Haplodiplö̈disation par Androgenèse In Vitro chez une Céréale Autogame: Triticum aestivum $L$. Thèse de doctorat d'état, Université de Paris XI, Orsay, France (n² 2902) $269 p$

Picard E., Parisot C., Blanchard P., Brabant P., Causse M., Doussinault G., Trottet M. \& Rousset M. (1988) Comparison of the doubled haploid method with other breeding procedures in wheat (Triticum aestivum) when applied to populations. In: Proceedings of the 7th International Wheat Genetics Symposium (Miller
T.E. \& Koebner R.M.D. eds), Cambridge, England, $1155-1159$

Scheinberg E. (1966) The sampling variance of the correlation coefficients estimated in genetic experiments. Biometrics 22, 187-191

Sneep J. (1976) Selection for yield in early generation of self fertilizing crops. Euphytica 26, 27-30

Speckel D., Vincourt P., Azaïs J.M. \& Kobilinsky A. (1987) Etude de la compétition interparcellaire chez le Tournesol. Biom. Praxim. 27, 21-43

Weber W.E. (1984) Selection in early generations. In : Efficiency in Plant Breeding, Proccedings of the 10th Congress of Eucarpia, (Lange W., Zeven A.C. \& Hogenboom N.G. eds), Pudoc, Wageningen, 72-81 